\title{
All you need is love. \\ Ciudadanía, género y educación en los debates del primer socialismo argentino*
}

\begin{abstract}
Marina Becerra ${ }^{* *}$
Resumo

En este trabajo presento un análisis de las estrategias socialistas para la producción de una ciudadanía moderna, en el período fundacional del socialismo (1896-1910), coincidente con el momento de sistematización de la escolarización básica en Argentina. El estudio de aquellos debates socialistas reviste especial interés para las problemáticas de género, puesto que el socialismo constituye una de las formaciones políticas que en Argentina defendió activamente la emancipación femenina e impulsó muy tempranamente la producción de una ciudadanía moderna para ambos sexos.
\end{abstract}

Palavras-chave: Socialismo, Género, Ciudadanía, Educación, Política.

\footnotetext{
" Recebido para publicação em março de 2005, aceito em novembro de 2005.

*** Instituto Interdisciplinario de Estudios de Género-Facultad de Filosofía y Letras- Universidad de Buenos Aires/CONICET. Doctoranda en el Programa de Doctorado en Ciencias Sociales (UBA).marinabbecerra@yahoo.com.ar
} 
All you need is love

All you Need is Love.

Citizenship, Gender and Education Inside the Debates of the First

Argentine Socialism

\begin{abstract}
This work pretends to analyze the socialist strategies for the production of a modern citizenship, during the socialism foundational period (1896-1910), coincident with the moment of basic scholarship in Argentine. The investigations about those socialists debates line a special interest for the gender problems because the socialism is one of the political formations that in Argentine strongly defended the feminine emancipation and very early impelled the production of a modern citizenship for both sexes.
\end{abstract}

Key Words: Socialism, Gender, Citizenship, Education, Policy. 
Marina Becerra

(...) esta obra del divorcio es santa, porque va a redimir de una servidumbre a millares de desgraciadas, porque es una campaña en pro de la redención de muchas personas atadas por vínculos que no nacen del corazón, porque importa una campaña en favor de todos aquellos que gimen en una de las servidumbres que es tal vez la peor de las servidumbres, funesta para el individuo y para el estado: la servidumbre sexual. (Del Valle Iberlucea, 1919:153-207)

(...) la educación de la infancia no puede ser dejada a la sola influencia del Estado, será una obra armoniosa debida a la acción de todos los que la aman. (Alicia Moreau, en Revista Socialista Internacional, 15/02/1909)

\section{Introducción}

En este trabajo presento un análisis de las estrategias políticas del socialismo en su período fundacional ${ }^{1}$, coincidente con el momento de sistematización de la escolarización básica en Argentina. El estudio de aquel socialismo reviste especial interés para las problemáticas de género, puesto que se trata de una de las formaciones políticas que en Argentina defendió activamente la emancipación femenina e impulsó muy tempranamente la producción de una ciudadanía moderna para ambos sexos. Estas luchas se libraron desde el Parlamento (con proyectos de sufragio universal, o de reforma del código penal incluyendo por primera vez la no penalización del aborto en determinados $\operatorname{casos}^{2}$,

\footnotetext{
1 Se puede identificar de tal modo al período extendido entre la constitución del Partido Socialista Argentino (1896) hasta 1910. En este período el partido impulsó diversas iniciativas culturales, tanto de formación de trabajadores como de sus hijos, tanto a través de instituciones formales (como escuelas) así como de otras no formales (como universidades populares). Asimismo, comenzó a participar activamente en la vida política argentina, logrando incorporar al parlamento el primer diputado socialista de América Latina, Dr. Alfredo Palacios, en el año 1904.

2 El "Proyecto de Código Penal en el Senado" salió de la Comisión de Códigos del Senado en 1919, incorporando al proyecto original el tema del aborto. Allí, el primer senador socialista de Latinoamérica, Enrique Del Valle Iberlucea,
} 
All you need is love

proyectos de reforma del código civil $^{3}$, etc) como desde la sociedad civil: tales fueron las múltiples propuestas de política educativa, sostenidas particularmente por el grupo de mujeres socialistas (Alicia Moreau, Justa Burgos, Pascuala Cueto, Mercedes Salaberry, María L. de Spada, Mariana Chertcoff, Fenia Chertcoff, Sara Justo, Raquel Caamaña, Raquel Messina, etc) algunas de ellas organizadas en un Centro propio (el Centro Socialista Femenino, cuya secretaria general era Fenia Chertcoff, quien había patrocinado la Liga por la Educación Laica). Asimismo, intelectuales socialistas que lucharon por la causa femenina -más bien olvidados - como Enrique Del Valle Iberlucea, Manuel Meyer Gonzalez, Bernardo Irurzun, defendieron también activamente los derechos de las mujeres ${ }^{4}$ ligados a la prioritaria tarea pedagógica que debía asumir el partido:

proponía muy tempranamente, la despenalización del aborto no sólo con fines eugenésicos sino también por los llamados "motivos sentimentales", esto es, en cualquier caso de violación. Del Valle explicaba que el problema del aborto se planteó en Europa en la guerra mundial "con motivo de las violaciones de que fueran víctimas numerosas mujeres belgas por soldados ebrios, desenfrenados o criminales". Ver Revista de Criminología, Psiquiatría y Medicina Legal, tomo 7, Bs. As., 1920:29-101.

3 Del Valle Iberlucea presentó en el Senado, en 1918, un proyecto que establecía la igualdad jurídica de la mujer, incluyendo la argumentación en favor del divorcio. Sin embargo, desde años atrás, y aún antes del primer debate sobre el tema en el Parlamento (20/07/1902), el joven Del Valle planteaba en su tesis doctoral para obtener el título de Doctor en Jurisprudencia en la UBA: "La mujer casada debe tener derecho, con absoluta prescindencia del marido, a los bienes que obtenga con su trabajo o la industria. En este sentido deberá reformarse el título correspondiente de nuestro Código Civil". En aquella tesis también planteaba ya la necesidad de incorporar el divorcio al código civil. Ver Del Valle Iberlucea, 10/06/1902:199 y 200.

4 Para sintetizar, a lo largo de este trabajo me refiero al "grupo de mujeres socialistas" que luchaban por sus derechos como ciudadanas y por la educación popular, pertenecientes en su mayoría a los centros socialistas femeninos. Sin embargo, es importante aclarar que estoy incluyendo en dicho grupo a estas voces masculinas que, junto a aquellas mujeres, han luchado activamente y desde múltiples posiciones por la emancipación femenina. 
Marina Becerra

Declaración de Principios del PSA. Programa Mínimo (...) Reforma de la Enseñanza: a) instrucción laica, gratuita y obligatoria para todos los niños hasta 14 años, b) Sostenimiento de los niños que frecuentan las escuelas, por los poderes públicos, c) Obligación de los municipios o del gobierno de establecer escuelas nocturnas para adultos, siempre que las soliciten cuarenta, sin excepción de sexo (...) (La Vanguardia 5 , 07-01-1905; cursiva mía)

Cabe señalar que en los primeros años del siglo XX la demanda de educación para ambos sexos, es altamente original y significativa, uno de los nudos fuertes de las luchas de los socialistas (así como el mencionado y desconocido planteo de Del Valle Iberlucea sobre la despenalización del aborto). Existía entre ellos una visible relación entre educación y ciudadanía, y también una relación - quizá menos visible - entre educación, ciudadanía y género, ya que la proyectada "elevación cultural de los trabajadores" (que se realizaría mediante la fundación de instituciones populares de cultura, como escuelas socialistas, entre otras) contribuiría en la formación de una conciencia ciudadana. La ecuación era la siguiente: a mayor educación correspondería mayor conciencia de los derechos de los trabajadores, tanto de los hombres como de las mujeres:

\footnotetext{
${ }^{5}$ La principal fuente analizada aquí es el semanario (luego diario) La Vanguardia, ya que se trata del órgano de prensa oficial del partido socialista argentino. El formato hemerográfico implica mayor circulación social (que el formato "libro"), lo cual le otorga un valor particular en tanto órgano decisivo de enunciación colectiva, así como medio de producción de una identidad común. Además, he complementado la revisión con revistas, cuyo objetivo es debatir en un campo intelectual algunos problemas de interés para el socialismo y las mujeres, con numerosos artículos no sólo de militantes, sino también de adherentes, aunque con una llegada más limitada a determinados círculos ilustrados que La Vanguardia. En este sentido, he revisado la Revista Socialista Internacional (1906-1909) así como la Revista Humanidad Nueva (1910-1918). Ambas revistas fueron fundadas por Enrique Del Valle Iberlucea, junto a Alicia Moreau.
} 
All you need is love

Trabajadores: la emancipación de nuestra clase necesita el concurso, no sólo de los obreros varones, sino de las mujeres proletarias, más explotadas aún que nosotros. Procuremos, por tanto, atraerlas a las filas del socialismo y no desperdiciemos ocasión de organizarlas por oficios. ( $L a$ Vanguardia, 13-05-1905)

Este párrafo da cuenta de esta cadena, incluyendo allí otro elemento: el paternalismo no sólo de la vanguardia iluminada, sino de la ficción moderna del individuo (masculino), estructuras de sentimiento ${ }^{6}$ bien compatibles en el molde iluminista propio de los socialismos de la época.

Pero los reclamos de aquellas mujeres por la ciudadanía se inscribían en una lucha por la igualación de los derechos civiles y políticos ${ }^{7}$ de las mujeres respecto a los hombres, en contra de la inferioridad jurídica de la mujer en la que se encontraba sometida. En este sentido, el concepto de ciudadanía implicado en estas luchas era neutral desde el punto de vista del género, esto es, tenía un sesgo claramente universalista. Esto significa que no luchaban por una posición diferencial específica según el género (en tanto mujeres) sino que, por el contrario, luchaban por la inclusión de las mujeres con iguales derechos que los hombres, tanto en la esfera pública como en la privada - votar, divorciarse, disponer de bienes y salario propios, ejercer su profesión, realizar acciones judiciales, educarse, etc.

Por otra parte, la conceptualización de la división entre esferas pública y privada como problema eminentemente político es bastante reciente. Es probable, además, que esta formulación haya sido posible después del aumento significativo de igualdad civil, debido precisamente a las luchas de las mujeres en el pasado por la inclusión con iguales derechos que los hombres. (Pateman, 1995)

${ }^{6}$ He tomado este concepto de Williams, 2000.

7 Aunque en ocasiones se demandaba el sufragio femenino "por etapas". Ver Barrancos, 2004. 
A continuación analizaré estos intentos de producción de una ciudadanía moderna por parte del primer socialismo, a partir de los debates entre aquellos hombres y mujeres sobre el problema de la integración social que la escuela - institución moderna por excelência - posibilitaba como ningún otro espacio obligatorio y legítimo de sociabilidad.

\section{En la búsqueda del sentimiento perdido}

Todo hombre o mujer debe ser una célula activa del organismo social, dedicando su actividad a una sola función (educador o médico, panadero o escritor, etc) para hacerse especialista y hábil en ella, toda mujer u hombre debe unirse a las otras células (hombres o mujeres) que desempeñan la misma función (todos los maestros, todos los médicos, todos los panaderos, todos los escritores, etc) para formar órganos robustos (congresos, gremios, etc) capaces de intensificar a la mayor potencia, en el juego armónico de todos, el bienestar y la riqueza del organismo (la humanidad). Trabajar y asociarse son los deberes cardinales del individuo en la colmena humana. Los que trabajan se llaman obreros (intelectuales o manuales), los que no trabajan, o dedican su actividad a ocupaciones improductivas para la colmena, se llaman zánganos (patrones, militares, religiosos, rentistas, ladrones, políticos, muchos funcionarios y empleados, etc). (Gonzalez, 1902) ${ }^{8}$

\footnotetext{
8 La Revista de Educación, fue fundada en 1891 y dirigida en la ciudad de La Plata por Manuel Meyer Gonzalez. Este olvidado maestro y militante socialista fue también co-fundador del primer centro socialista de La Plata, en enero de 1901, así como de una de las primeras escuelas socialistas del país, y también de la Asociación de Maestros de la Provincia de Buenos Aires en el año 1900. La Revista de Educación -cuyo subtítulo a partir de la constitución de la Asociación de Maestros fue Organo gremial del Magisterio de la provincia de Buenos Airesconstituye una de las fuentes -muy poco explorada- más interesantes para los problemas aquí planteados. Se trata de un espacio amplio de discusión entre docentes e intelectuales dedicados a analizar problemas de educación, y con una fuerte orientación socialista, abarca un espectro de educadores liberales asociados de diversas formas en las iniciativas de educación popular, revelando
} 


\section{All you need is love}

Hace más de 100 años, la actual pregunta por la otredad constitutiva $^{9}$ era para hombres y mujeres socialistas altamente problemática, puesto que luchaban por integrarse en una sociedad que se constituía sobre la heterogeneidad producto de los acelerados procesos de inmigración. ${ }^{10}$ ¿Qué propuestas de integración social tenían aquellos socialistas frente al discurso estatal que fundaba la integración en la anulación de las diferencias culturales en pos de la producción de una nación homogénea ${ }^{11}$, construyendo sobre esa base al modelo de ciudadano argentino (es decir) masculino? Si la propuesta de integración de la nueva política pedagógica estatal - el

por ello aspectos que las publicaciones estrictamente socialistas no abordan. Asimismo, la circulación de la misma anuncia un recorrido más amplio que las publicaciones partidarias.

9 En este punto, no me estoy refiriendo al nivel de la otredad constitutiva del lenguaje como género discursivo que me antecede, sino que me refiero específicamente al nivel del otro semejante, en interacción con el cual se constituyen los sujetos.

${ }^{10}$ Sobre los procesos de inmigración masiva en el período, se puede consultar Cibotti, Ema. Del habitante al ciudadano: la condición del inmigrante. (Zaida Lobato, 2000)

${ }^{11}$ Un ejemplo de esta urgente y necesaria producción de ciudadanos está dado por la ley número 4874 (llamada Ley Lainez) del año 1905, momento bisagra del sistema educativo argentino, en tanto establecía mayor presupuesto para la edificación de escuelas primarias, ampliando la zona de influencia del Estado nacional en las provincias (donde antes no tenía ingerencia) a través de la fundación masiva de escuelas nacionales. En este sentido, la Ley Lainez constituye uno de los intentos estatales más sólidos de producción hegemónica en materia educativa en el período. Curiosamente no se dispone de investigación alguna acerca de esta ley. La hipótesis aquí formulada parte de la constatación -a partir de las páginas de La Vanguardia- de un mayor interés estatal por la construcción de escuelas primarias luego de 1905, del mayor presupuesto destinado a educación, etc. Esta percepción me llevó al análisis de las estadísticas del sistema educativo en el período (Gandulfo, 1991) confirmando allí las cifras publicadas por la prensa socialista de principios de siglo. De hecho, hacia 1909 ya funcionaban en las provincias 700 escuelas dependientes del Estado nacional. Para ver la recepción realizada por los socialistas de la propuesta del senador Lainez, véase La Vanguardia (en adelante LV 08-09-1905. 
nacionalismo - se fundaba en la homogeneización cultural, ¿cómo pensaban los socialistas articular estas diferencias en la producción de una ciudadanía democrática?. Si aquella oferta estatal de producción de ciudadanía moderna se estructuraba sobre la incipiente igualdad civil (reino reservado al sexo - masculino) montada en la desigualdad privada (espacio del género femenino, es decir, de las diferencias) ¿qué propuestas tenían los socialistas frente a estas aparentes contradicciones? En tal contexto ¿cómo conceptualizaban los socialistas la diferencia sexual en la producción de ciudadanía?

La búsqueda de formas corporativas de organización social, así como de la especialización de funciones para la "mayor potencia del organismo social", donde la importancia de la especialización derivada de la división sexual y social del trabajo deviene de su poder cohesionador - fuente de solidaridad social en tanto generadora de "órganos robustos" como dice el párrafo citado - aparece con suma frecuencia en los relatos de los primeros socialistas argentinos. Esta versión local, coetánea de las ideas durkheimianas del orden social, también se expresa en las propuestas socialistas para la libre dirección de las escuelas primarias, donde, asociada a la defensa de las formas corporativas, aparece la conocida fórmula sarmientina de plantear la lucha por la educación popular: que la administración sea popular (esto es, dejada a la libre "opinión" de los consejos escolares) y no oficial. En otras palabras, dejando la dirección de la educación pública en manos de "corporaciones orgánicas", se fortalecerían los lazos de la sociedad civil, produciendo de este modo ciudadanos solidarios entre sí. En este sentido, parecía posible establecer relaciones sociales en las cuales las diferencias sexuales podrían articularse en la sociedad civil precisamente en tanto diferencias de género, culturales. En términos saintsimonianos - tan familiares a aquellos socialistas - era deseable que la "administración de las cosas" fuera desplazando con su fuerza civilizatoria al "sistema de gobierno" que intentaba centralizar cada vez más funciones en su seno descalificando de 
ese modo la potencia de la organización corporativa que podría surgir desde la sociedad civil. Sin embargo, no todos los socialistas coincidían en esta forma de pensar la tensión entre particularidad y universalidad, donde las diferentes "células" particulares podrían ser jerárquicamente iguales (un hombre no es mejor que una mujer, un maestro no es mejor que un panadero, etc) pero diferentes en sus funciones. Y sería precisamente esa diferencia, esa incompletud, lo que daría riqueza, puesto que generaría relaciones de solidaridad (a partir de la necesidad que cada uno tiene de los otros).

De ese modo, el sentimiento de solidaridad llevaría a una sólida integración social entre hombres y mujeres. Esa integración sería, a su vez, fundamento de una ciudadanía moderna. El problema entre los socialistas era si esa ciudadanía sería universal, partiendo de un supuesto de igualdad entre todos los individuos (considerados, por ello, como individuos abstractos, bajo el modelo de individuo moderno: masculino), entre hombres y mujeres, o si esa ciudadanía se podría fundar en las capacidades diferenciales de los diversos individuos concretos (sexuados) como sugiere la pluma de Manuel Meyer González. En la misma línea, Enrique Del Valle Iberlucea sostenía en una conferencia dada en el Centro Socialista Femenino en 1902:

(...) hay que confesarlo, hay que tener el valor de confesarlo, al hombre, pertenezca a la burguesía o al proletariado, le falta la conciencia de que él no es superior a ella, de que según los datos de la antropología y de la psicología, él es un ser diferente pero no superior a la mujer. (Grandes Aplausos). Es una dolorosa verdad (...) que el hombre ha ejercido siempre esta coerción física y esta coerción moral sobre la mujer (...) Permitidme que haga constar que el hombre, legislador siempre, ha traducido en la ley su predominio y su absolutismo, en todas las épocas y en todos los pueblos. (Del Valle Iberlucea, 1919;153-207) 
Marina Becerra

El joven Del Valle, recién egresado de la Facultad de Derecho, tenía ya la sensibilidad y lucidez de percibir que la inclusión de la mujer en la vida política era una lucha que debía darse también en la letra. ${ }^{12}$

\section{Debates socialistas}

La vía educativa constituía uno de los puntos centrales en esta necesaria y urgente producción de ciudadanos tanto en las políticas estatales ${ }^{13}$ como en políticas alternativas. En efecto, la tarea de democratizar la vida política que el socialismo se había asignado se fundaba, en gran medida, en la educación de los trabajadores y de sus hijos, constituyendo por ello un eje fundamental en la producción de una ciudadanía incluyente para hombres y mujeres. En este marco, los socialistas argentinos generaron importantes experiencias educativas con gran cantidad de alumnos en numerosos barrios de la Capital y en varios pueblos de la provincia de Buenos Aires ${ }^{14}$, con reconocimiento

${ }^{12}$ Tarea a la cual se abocó desde su banca en el Senado de la Nación desde 1913, hasta su desafuero en 1921, por su posición en favor de la revolución rusa, apartándose así de la posición oficial del Partido Socialista (el grupo de J.B. Justo y Nicolás Repetto, los intelectuales partidarios "de primera línea").

${ }^{13}$ Sobre la creciente feminización del magisterio en los primeros años del siglo XX (al compás del avance estatal en educación), y la educación de la mujer educadora de los futuros ciudadanos que el Estado necesitaba en la producción de la nación, puede consultarse Yannoulas, Silvia. La educación de la mujer educadora en la construcción de la educación nacional. (Cadernos de Textos, $\mathrm{n}^{\circ}$ 4, Doutorado Conjunto FLACSO/UnB, 1993) Un análisis del original pensamiento de una de aquellas mujeres socialistas, Raquel Camaña, en sus críticas a la preponderancia femenina en el magisterio, puede encontrarse en Morgade, Graciela. Quiénes fueron las primeras maestras. (IICE, FFyL-UBA, Miño y Dávila, Buenos Aires, 1992:52-60)

${ }^{14}$ No todas las escuelas tuvieron la misma continuidad, ni todas ellas contaron con la misma oferta de cursos (algunas funcionaban con pocos grados). Entre las escuelas primarias para niños, hemos encontrado para el período estudiado: Escuela Internacional (1900); La Escuela del Pueblo de La Plata (1900, impulsada por iniciativa de Manuel Meyer Gonzalez, director de la Revista de Educación); La Escuela del Pueblo (1901); Escuela Mixta de La Banda (abierta 


\section{All you need is love}

oficial de los títulos expedidos en las más importantes de sus escuelas primarias, cuyos programas, además, se homologaban a los de las escuelas estatales. Alli participaron importantes grupos socialistas ligados a actividades gremiales y docentes ( $\mathrm{La}$ Vanguardia, 05-08-1905) pero no sólo a ellas, ni se trató sólo de militantes orgánicos del partido socialista. ${ }^{15}$ Es importante señalar que no se trató de algunas pocas experiencias esporádicas, sino que en los albores del siglo, la fundación de escuelas populares constituyó un esfuerzo ampliamente compartido entre los (y especialmente las) socialistas ligados a funciones intelectuales -en tanto creadores de sentidos. (Gramsci, 1962)

Asimismo, se trató de espacios de experimentación educativa, resonancia de los movimientos internacionales de renovación de la escuela, en tanto los socialistas sostenían que "la educación burguesa y poco práctica de las escuelas fiscales"16 (sic) constituía un obstáculo que debía ser "desterrado" de las escuelas. Pero estas experiencias convivieron conflictivamente con las tendencias (internas y externas al partido socialista) que, mirándose en el espejo francés, sostenían que debía ser el Estado el responsable único de la educación. Estos debates se producían en los intentos socialistas por generar las condiciones para una ciudadanía moderna, que la escuela estatal facilitaba en su avance

en 1903); Escuela Laica de Morón (fundada en 1904); Escuela Laica de La Boca (promovida por el Centro Socialista Femenino, y fundada en 1906); Escuela Laica de Lanús (abierta en 1906, impulsada por la Sociedad de Educación Laica de Lanús); Escuela Laica "Bernardino Rivadavia" de Lobos (1908); Escuela "La Colmena" de La Plata (fundada en 1908, a iniciativa de Justa Burgos Meyer, también impulsora del Centro Socialista Femenino de La Plata); Escuela de Unión Colegiales (1908); Escuela Laica de Pergamino (1909). Para otros detalles (como la duración de las escuelas, así como de las características del funcionamiento) puede consultarse el trabajo de Barrancos, 1991.

${ }^{15}$ También colaboraron en el sostenimiento de estas escuelas, adherentes al partido, así como intelectuales del campo de la educación estatal, y figuras del anarquismo.

${ }^{16}$ Las escuelas dependientes del Estado se denominaban también "escuelas fiscales". 
Marina Becerra

integrador, volviéndose por ello mismo, mejorable y hasta deseable ${ }^{17}$ para varones y mujeres.

Por otra parte, la propuesta de integración hegemónica en los tiempos del Centenario se basaba en el nacionalismo, y era precisamente este motivo lo que impulsaba el objetivo político de masificar la educación. Frente a esta propuesta, el PS se proponía producir algún modo de integración social distinto al nacionalismo hegemónico, que ligara la crecientemente conflictiva cuestión social con la cuestión nacional. En este sentido, es sabido que los intelectuales han jugado históricamente - $y$ desde muy temprano un rol central en la articulación del vínculo entre cultura ("fuerzas morales": educación, historia ${ }^{18}$, tradición, en términos de los intelectuales de fines del siglo XVIII y principios del XIX) y nación, en el sentido de asumir una posición de mediación donde la cultura aparece como un elemento decisivo en la imaginación de una comunidad nacional. Quizá era esta articulación la que muchos socialistas (ligados a sectores liberales reformistas de la élite intelectual y en particular, mujeres) intentaban llevar a cabo con la creación de escuelas propias, asumiendo en la práctica estas tareas de mediación a través de la educación de los niños, como uno de los temas fundamentales del partido. Fue esta dimensión simbólica de la identidad política la que, según Aricó (1999), aquel socialismo no pudo contemplar por haber establecido una relación de transparencia entre las posiciones en la economía y en la política. Pero si bien fue ésta la concepción hegemónica en el partido socialista (planteada por Juan B. Justo ${ }^{19}$

\footnotetext{
${ }^{17}$ Este tema fue trabajado en mi Tesis de Maestría a partir del análisis de la relación entre el avance estatal en el proceso de producción hegemónica en el momento de sistematización de la educación básica y los desplazamientos de las estrategias político educativas de los primeros socialistas. Ver Becerra, 2003.

${ }^{18}$ Cabe aclarar que el término historia aquí se refiere a los usos y costumbres, a la vida privada, a las relaciones con las mujeres, a la moral, etc. Ver Chabod, 1997.

19 Juan B. Justo (1865-1928) fue co-fundador del Partido Socialista Argentino en 1896, y uno de sus intelectuales y dirigentes políticos más destacados. Además
} 


\section{All you need is love}

y el núcleo dirigente formado en torno suyo) nos encontramos con otras voces que concebían la acción política desde interpelaciones colectivas a partir de elementos no necesariamente racionales, sobre referentes tales como "los sentimientos, la imaginación, la música, la pasión" (sic). Al respecto, las proposiciones realizadas por el Centro Socialista Femenino, el Centro Socialista de La Banda (provincia de Santiago del Estero), y el de La Plata, entre otros, ilustran esta voluntad de producir una identificación fundada en un sentimiento de solidaridad mediante la creación de escuelas socialistas:

El Congreso resuelve: Que vería con agrado que todos los centros organizados de la República aceleraran el establecimiento de escuelas laicas para obreros (...) Considerando: 1. que de la instrucción de las fuerzas obreras depende el adelanto de las organizaciones proletarias, 2. que es un deber de los gobiernos progresistas la educación civilizadora del pueblo para que entre en el concierto de las nuevas ideas, 3 . que los representantes del PS están en el deber de concurrir con su inteligencia a propagar el sentimiento de solidaridad, resuelve: Pedir a los diputados del partido proyecten leyes subvencionando las escuelas que los centros obreros sostienen con el esfuerzo de sus asociados (...). Un consejo o comisión permanente y especial se encargará de estudiar y solucionar el problema educacional de la clase trabajadora proporcionando a los obreros y a sus hijos aquellos medios de desenvolvimiento integral y armónico de sus facultades que el Estado burgués no les da o les ofrece incompletos (...). Este consejo se preocupará preferentemente de la fundación de escuelas elementales diurnas, nocturnas y dominicales, en los barrios y poblaciones fabriles, y tendrá la dirección técnica de estas

de diputado (desde 1912) y luego senador nacional (desde 1924) fue también el primer traductor al español de El Capital, de Carlos Marx, obra publicada en Madrid en 1898. Asimismo, participó de las reuniones de la Segunda Internacional en Berna y en Ámsterdam como representante del socialismo sudamericano. 
Marina Becerra

instituciones, lo mismo que de toda obra de cultura obrera ya existente o que se fundare en lo sucesivo (...) Lo compondrán 7 compañeros que pertenezcan, en cuanto sea posible, al gremio de profesores o maestros de ambos sexos (...). (LV, 20-03-06; cursiva mía)

La propuesta de estos socialistas para la creación de un concejo de Cultura Obrera compuesto por maestros de ambos sexos, da cuenta de la percepción del mencionado rol de mediadores culturales de los intelectuales, así como de la presencia explícitamente necesaria de hombres y mujeres, en términos de igualdad. Este punto es particularmente singular, puesto que evita los lugares tradicionales donde sólo los hombres - considerados como los únicos sujetos racionales - estarían en condiciones de decidir sobre las iniciativas político-culturales, así como el que asocia a las mujeres con el cuidado de los niños y el magisterio. Por el contrario, plantea las posiciones de hombres y mujeres en términos de igualdad (de posibilidades, de derechos) asumiendo así uno de los elementos básicos del modelo universalista mencionado al inicio del trabajo.

Pero además, complejizando este modelo universalista de ciudadanía, la existencia de escuelas socialistas puede interpretarse también como un intento de realizar estas tareas de mediación hegemónica donde la búsqueda de un elemento cohesionador se percibe más como sentimiento - de solidaridadque como identificación racional. Esta centralidad de la dimensión simbólica en la producción de ciudadanía constituye un tema epocal en sentido bajtiniano. (Voloshinov e Bajtin, 1992) En efecto, esta pregunta clave de la modernidad - tensión entre ruptura $e$ integración ${ }^{20}$ vinculada a la sociabilidad que la escuela garantizaba

${ }^{20}$ Esta tensión fue analizada por Durkheim también a partir de la propuesta de reconstrucción del sentimiento de solidaridad en las sociedades crecientemente complejas. Y este tema, a través de la lectura soreliana de la obra de Durkheim, fue retomado años más tarde por Gramsci. No es extraño que este drama de la modernidad apareciera también atravesando la morada del socialismo argentino, 
All you need is love

como ninguna otra institución, se debatía entre los socialistas originando dos identidades políticas en tensión, vinculadas a los diversos modos de imaginar una ciudadanía moderna. Estas diferencias podrían fundarse, a su vez, en distintas concepciones de la política.

Por un lado, la decisión finalmente adoptada $^{21}$, si bien confluía con el objetivo de política educativa estatal en cuanto a la masificación de la educación, pasaba por alto el hecho de que la educación estatal debía ser nacionalista porque era ésa precisamente su principal función política. Por otro lado, mantener escuelas propias ponía de relieve el problema estructural de la integración del socialismo en la nación argentina. Una base discursiva de esta certeza socialista estaba constituida por el cuidadoso reemplazo de las que empezaron a llamarse escuelas populares o laicas en lugar de las llamadas escuelas socialistas (hasta 1901) en tanto los socialistas consideraban que las últimas presentaban ribetes sectarios que las primeras no tenían. Así se podrían integrar en la escuela docentes y niños de diferentes procedencias, aún cuando sus padres no fueran socialistas, y de ese modo, precisamente, el socialismo podría ir difundiéndose capilarmente $e$ integrándose lentamente en la vida social argentina, transformando a los niños en ciudadanos democráticos.

Esta posición, que apelaba al sentimiento de solidaridad como nota fundante de la identidad socialista y de la ciudadanía moderna, luchaba externa e internamente por la creación de escuelas propias. ${ }^{22}$ Lo "moderno" de la ciudadanía se asociaba

siendo el concepto de solidaridad una referencia significativa que informaba las teorías más disímiles sobre lo social desde fines del siglo XIX.

${ }^{21}$ En el IX Congreso del PS realizado en diciembre de 1910 se decidió abandonar las iniciativas educativas propias ( $L V, 26$ y $27 / 12 / 1910)$

22 "Con placer tomamos nota del interés con que en las cabezas del pueblo empiezan a germinar como una preocupación y un programa los problemas de la escuela. El PSA que, aunque recién aparejado para una figuración relevante en las luchas políticas del país, acaba de celebrar su 5to Congreso en Bs As, ha registrado en las actas de sus secciones los siguientes propósitos de acción y 
aquí con un sentimiento (de solidaridad) a diferencia de la primera posición, donde el significante "modernidad" se articulaba al modelo (masculino) racional. Pero la fundación de escuelas propias, impulsada en especial por las mujeres socialistas, no se concebía meramente como una forma complementaria de la educación estatal, sino que aparecía también como un gesto frente a las políticas de inclusión estatales, en tanto uno de los puntos de la crítica se vinculaba con la orientación "burguesa y poco práctica" de las escuelas fiscales. En otros términos, se confrontaban con las pedagogías "abstractas" propias de la educación humanista tradicional, que producía eficazmente ciudadanos masculinos, los únicos individuos "aptos" para la vida pública, plenamente racionales y dueños de sí. En este sentido, constituían experiencias educativas ideológicamente diferentes en tres sentidos. En primer lugar, se trataba de escuelas despojadas de religión, así como, en segundo término, de los elementos xenófobos y racistas del nacionalismo hegemónico (que, según aquellos socialistas, justificaba además el orden capitalista) en las escuelas estatales. Por último, las escuelas socialistas serían instituciones modernas, de orientación práctica, a diferencia de las escuelas del Estado. Todavía en 1909 Alicia Moreau defendía esta posición:

La escuela es, por último, la escuela del Estado, que es la sanción y el sostenimiento del régimen social actual, no puede por lo mismo ir contra sentimientos $e$ ideas que hacen posible este régimen. Debe, por lo tanto, aplacar el sentmiento de rebeldía, hacer encontrar bueno lo injusto, y justificar las desigualdades sociales (...) Es necesario renovar la escuela (...) Ese sentimiento nuevo [la solidaridad], tal

propaganda: sostenimiento de los niños que frecuentan las escuelas por los poderes públicos y creación de escuelas populares bajo un plan especial a fin de sustraer a la educación burguesa y poco práctica de las escuelas del estado el mayor número posible de niños proletarios (...) es una verdad establecida por la estadística que hay un $59 \%$ de analfabetos en la población general de la república". (Revista de Educación, 1/7/03) 
All you need is love

vez hijo del ferrocarril y del telégrafo, centuplica la acción, y cuando él impere, será un hecho el lema de la Escuela Renovada: 'la educación de la infancia no puede ser dejada a la sola influencia del Estado, será una obra armoniosa debida a la acción de todos los que la aman. (Alicia Moreau, Revista Socialista Internacional, 15/02/09)

Aquí aparece nuevamente el sentimiento de solidaridad, hijo nuevo y bueno de la modernidad capitalista, germen de su propia destrucción. Además, ese sentimiento "centuplica la acción", es decir que allí donde existe, se fortalecen los lazos sociales produciendo acciones de mayor potencia, aún siendo hijo de la extrema especialización que la división del trabajo genera en esa fase de la producción. Por otra parte, para aquellas mujeres socialistas era preciso realizar una reforma curricular de la educación estatal, pero ello era interpretado como ideológicamente contrario a las necesidades de reproducción social del capitalismo. Por lo tanto, el camino que quedaba consistía en sostener escuelas socialistas bajo el impulso de la "renovación" escolar, lo cual implicaba, entre otras cosas, que las más importantes de dichas escuelas daban clases al aire libre y tenían una definida orientación práctica. Implicaba, además, que sólo aquellos que "amaran" la educación de los niños, podrían llevar a cabo semejante tarea. Vuelve a estar presente entonces la dimensión del sentimiento, ausente en cambio en las formulaciones más racionalistas características del entorno de Justo - grupo hegemónico -que decidió abandonar en 1910 el sostenimiento de las escuelas socialistas.

\section{La escuela popular laica de Morón}

El análisis de la experiencia educativa más importante del socialismo - tal como definía Nicolás Repetto a la Escuela de 
Morón $^{23}$ en el informe al IX Congreso del PS - pone de relieve dos temas centrales. Por un lado, el primordial objetivo de democratización de la sociedad, vinculado precisamente a esa forma de aprender "práctica" de la escuela moderna y renovada que propone Alicia Moreau. Por otro lado, la articulación existente entre el socialismo, la Asociación de Maestros, y algunas autoridades educativas del Estado. Esta articulación es comprensible precisamente en el marco del primer objetivo señalado: aquello que separaba las aguas no era la pertenencia partidaria, sino la búsqueda - o no - de la ampliación de la ciudadanía. A continuación puede verse una amplia articulación entre diferentes grupos, en defensa de la directora socialista de la escuela:

Pro Pascuala Cueto: Iniciada y auspiciada por la Sociedad Cosmopolita de Trabajadores tuvo lugar en Morón el domingo 13 una manifestación verdaderamente edificante y poco común en nuestro país. Las familias de las que fueron alumnas de la srta. Cueto, cuya exoneración nos ocupa editorialmente, y una masa compacta de vecinos, en que predominaba el elemento obrero, se congregaron en la plaza pública para protestar contra el inquisitorial castigo de la autoridad escolar y formular votos de cumplida reparación. Interpretando estos sentimientos hablaron la señora Clemencia $C$. Ceballos -antigua educacionista, hoy desligada de la escuela oficial pero que sigue prestando no menos eficaces servicios a la cultura popular como profesora y compañera de la srta. Cueto en la instrucción de los trabajadores- (...) el profesor Jorge Susini, que con su

${ }^{23} \mathrm{El}$ conflicto que dará origen a la escuela de Morón fue publicado en la Revista de Educación, agosto de 1903, números 7 y 8, año XI. Asimismo, apareció en La Vanguardia (30-01-04) en un artículo en primera plana sobre la corrupción de la Dirección General de Escuelas, del cuerpo de inspectores de la educación en la provincia de Buenos Aires, del comisionado escolar de Morón, del Consejo General de Escuelas, del ministro de gobierno y del gobernador de la provincia, ya que habían destituido a la directora de la escuela oficial, Pascuala Cueto mujer y socialista - para poner en su lugar al pariente de un inspector. 
All you need is love

colega el sr. Meyer Gonzalez representaba a la Comisión Central de la Asociación de Maestros y que puso en evidencia con entusiastas palabras la necesidad de la unión de todas las fuerzas docentes de la provincia (...) De las muchas adhesiones por escrito, señalamos la del sr. Víctor Mercante, director de la Escuela Normal de Mercedes, y la del Centro Socialista Femenino de Bs As (...). (Revista de Educación, agosto de 1903, nº 7 y 8 , año XI; cursiva mía)

Aquí observamos la particular convergencia entre vecinos obreros, antiguos docentes de la escuela oficial, maestros/as y militantes socialistas ${ }^{24}$, el conocido director de la exitosa Escuela Normal de Mercedes, y por supuesto, el Centro Socialista Femenino de Buenos Aires, siendo las mujeres, como señalaba anteriormente, una de las principales fuerzas comprometidas con el impulso y la defensa de escuelas socialistas, como se aprecia en la resolución propuesta ante el conflicto de la escuela oficial de Morón:

(...) Por iniciativa de la Sra. Justa Burgos Meyer, el Centro Socialista Femenino de Bs. As. se ha propuesto fundar en Morón una buena escuela particular frente a la oficial que dirigía la Srta. Cueto a quien se le entregará la dirección del nuevo establecimiento (...) Como los fondos que se requieren para esta fundación no han de ser exiguos y menos si se considera que el sostenimiento de la escuela exige un impulso poderoso para que sus beneficios alcancen gratuitamente a los niños pobres que fueron siempre los predilectos de la Srta. Cueto y que no deben ser excluidos de esta escuela verdaderamente popular (...) [se pide colaboración voluntaria] Dirigirse a la Sra. Justa Burgos Meyer en esta administración, o a Fenia Cherkoff

${ }^{24}$ Existía colaboración manifiesta entre el gremio de maestros y el socialismo (Manuel Meyer Gonzalez había impulsado la fundación del Comité Socialista de La Plata, donde militaba orgánicamente, mientras dirigía la Revista de Educación), aunque hacia 1907 esta unión comenzaba a disolverse. 
Marina Becerra

Repetto, Mexico 2070, Bs. As., o al Dr Angel Jimenez,

Morón. (Revista de Educación, agosto de 1903, nºs 7 y 8, año XI)

Por otra parte, los alumnos de la Escuela Popular de Morón ${ }^{25}$ debían anotarse en el Consejo Escolar para asistir a la escuela (La Vanguardia, 06-02-04), ya que tenía reconocimiento oficial. De modo que aún aquellos socialistas que - como el grupo que participaba también en el gremio docente, y especialmente las mujeres agrupadas en el Centro Socialista Femenino - promovían la fundación de escuelas propias como formas educativas ideológicamente diferentes a las escuelas estatales (educando a los niños y niñas a través de prácticas pedagógicas modernas que producirían ciudadanos democráticos) valoraban positivamente la credencial del Estado para legitimar su obra educativa. Y precisamente la orientación práctica de la escuela renovada era uno de los rasgos fundamentales de la Escuela de Morón:

[Sobre la Escuela de Morón] (...) Se han hecho excursiones a varias quintas y a las vías férreas (...) Se ha visitado un horno de ladrillo, un Acuarium. Se han observado animales, plantas, conversando, estudiando, coleccionando y dibujando luego en la Escuela lo visto (...) En las clases de agricultura se ha preparado la tierra, se ha sembrado y cosechado legumbres y flores. De esta manera, dice en su informe la directora "los niños adquieren conocimientos prácticos que dan, por cierto, mejores resultados que toda la teoría que se les inculca en las escuelas (...)" En el taller de costura se han confeccionado vestidos y ropa blanca. Se han realizado varias fiestas en que se ha combinado lo agradable con lo útil. Se han dado varias conferencias sobre la educación geográfica e higiene con proyecciones luminosas por la sra. G. De Coni, Dres. Nicolás Repetto,

${ }^{25}$ La escuela de Morón ofertaba en 1908, Jardín de Infantes, escuela primaria completa (1ero a 6to grado), los tres primeros años del Colegio Nacional, y clases nocturnas para trabajadores, además de incluir la capacitación regular de sus docentes. 
All you need is love

Pedro Torres (Inspector seccional de la capital), Angel

Gimenez (...). (LV, 27-05-05; cursiva mía)

La educación práctica se refería a un modo de aprender que se producía en el mismo trabajo, en el hacer cotidiano, vinculado a la producción de futuros ciudadanos socialistas. Esta forma de aprender era considerada distinta al modo de aprender abstracto, propio de las escuelas estatales. Ese modo "abstracto" que no les serviría a los obreros ${ }^{26}$ era el mismo que produciría a ese ciudadano abstracto y plenamente racional, modelo masculino que podría participar en la esfera pública precisamente por su capacidad de trascender el mundo de lo concreto y de las sensaciones, el mundo del cuerpo. En efecto, tal como dice el párrafo, en la combinación de "lo agradable con lo útil" aparece la mezcla, el desorden concreto de la sensación corporal con la razón. Porque así, desde la más tierna infancia, los futuros ciudadanos democráticos aprenderían en sus propias prácticas pedagógicas - luego políticas - la inigualable fuerza de lo social que se descubre a partir de la cooperación entre diferentes funciones o momentos del trabajo. En este sentido, se oponía la escuela laica, práctica, socialista, renovada y al aire libre, frente a la escuela burguesa, poco práctica, nacionalista e incluso religiosa, del Estado.

De esta manera, todos los socialistas coincidían en que una de las principales tareas a desempeñar por el partido se vinculaba con la producción de una ciudadanía moderna y democrática, universal: todos los individuos (extranjeros y nativos, hombres y mujeres, patrones y obreros) debían tener los mismos derechos. Aún aquella posición romántica que defendía la vía educativa propia, impulsada en particular por las mujeres socialistas convergía en puntos estratégicos, como es la democratización de la vida social, con otros grupos sociales que trabajaban desde el Estado por realizar reformas liberales (Zimmermann, 1995) en el

${ }^{26}$ Ver también La Vanguardia, 07/08-11-1910. 
campo educativo. En otros términos, intentaban resolver el problema de la ciudadanía a través de la inclusión en su propia particularidad, de otros particularismos. Esta estrategia de negociación da cuenta, al menos parcialmente, de cierta voluntad hegemónica por parte de este grupo. En este sentido, esta propuesta de ciudadanía consistía en la articulación de los principios de división del trabajo, solidaridad, amor y derechos universales (iguales derechos para hombres y mujeres).

La segunda posición, dominante entre los socialistas, respondía a la pregunta por la ciudadanía de un modo menos conflictivo en principio, ya que ésta se produciría con la asistencia de los hijos de los trabajadores a las escuelas - cada vez más laicas y más numerosas - del Estado. En cuanto al crecimiento del socialismo, éste no tendría asidero en sentimiento alguno, sino que a través de otras estrategias, como interpelaciones racionales, los trabajadores asumirían, a nivel de la conciencia, su posición de clase explotada, sumándose por ello mismo a las filas del socialismo. En este sentido, el problema señalado acerca de la pretendida relación de transparencia entre las posiciones en la economía y en la política, se podría vincular con esta estrategia de producción de una ciudadanía fundada en identificaciones de orden puramente racional. Esta posición era coherente con la creciente parlamentarización de la vida política del partido, donde se visualizaba la integración del socialismo a través de acuerdos con sectores reformistas liberales, que iban más allá de la cuestión educativa. Esta estrategia contemplaba también la incorporación de otros particularismos, pero no precisamente de elementos (como aquellos ligados a la simbología popular) provenientes de la "clase obrera" que el socialismo, paradójicamente, pretendía constituir y a la vez representar. Por el contrario, los elementos que incorporaba a su propia particularidad, se vinculaban con algunas definiciones básicas del proyecto educativo estatal (como la defensa del centralismo estatal en educación y la consiguiente extensión de la escolarización masiva) asumiendo en este sentido un modelo también universal de ciudadanía, pero a partir de los 


\section{All you need is love}

elementos más tradicionales de la teoría liberal (el hombre ciudadano: plenamente racional, abstracto). ${ }^{27}$ En fin, si existía en esta estrategia alguna voluntad hegemónica, la ampliación de sus definiciones en pos de una pretendida ciudadanía universal se vinculaba más con los elementos identificados racionalmente como democrático-liberales que sustentaban las políticas educativas estatales, que con elementos dispersos no necesariamente racionales de grupos de trabajadores que "deberían" asumir una identidad socialista esencial en función directa de su posición en la estructura económica. Atrapados en estas contradicciones y siendo ésta última la tendencia que hegemonizó finalmente en el partido, los socialistas no tuvieron la capacidad hegemónica de integrarse en base a elementos diversos

\footnotetext{
${ }^{27}$ Volviendo nuevamente a Francia -significativo modelo educativo para los socialistas argentinos- preguntas similares son formuladas por Agulhon (1994) en su análisis de las distintas lógicas existentes entre los republicanos franceses respecto del combate a la Iglesia: por un lado, la lógica liberal, racionalista y laica (presente en personajes como Jules Ferry) aconsejaba eliminar lo sagrado, lo místico, las figuraciones simbólicas del poder, y/o reducir estos elementos a la vida privada. Pero también existieron otros republicanos -y esto resulta particularmente interesante como sugerente analogía histórica para nuestro análisis- que veían que aún abolida la monarquía, el poder contrarrevolucionario de la Iglesia católica seguía siendo amenazador, a través de ritos, místicas, símbolos y representaciones: "De ahí la idea de oponer una religión a la religión, unos símbolos a los símbolos, de luchar en cierto modo sobre un mismo terreno (...)". Según el autor estos fenómenos de contrarreligiosidad en Francia fueron minoritarios ya que el hecho de que “(...) los defensores de las políticas del pasado tengan iglesias, rituales $e$ ídolos, es cosa natural; pero que los que propugnan un futuro racionalista opongan Iglesia a Iglesia, rito a rito e ídolo a ídolo, es una contradicción. Se desprecian más los viejos cultos si no se compite con ellos en su terreno, acostumbrando al pueblo a una política sin rituales, sin faramalla visual, austera, transparentada y fría. Tal vez era ésta la cuesta que estaba tomando el siglo XIX". Era la misma cuestión que estaban discutiendo los socialistas argentinos a principios del siglo XX: el problema de la romántica creencia en el sentimiento como elemento identificatorio opuesto a una anhelada (pero imposible) transparencia de lo político (propuesta por Justo) que tendría como supuesto la existencia de una política puramente racional. Ver Agulhon, 1994:267-269.
} 
de culturas populares, pues su posicionamiento racionalista de vanguardia iluminada se lo impedía. Aún así, es preciso destacar que la búsqueda de la ampliación de la ciudadanía, a través de la modernización de las arcaicas instituciones de la sociedad argentina, fue un tópico central reconocido - aunque con importantes diferencias en las acentuaciones y significados que esto tenía - por todos los hombres y las mujeres socialistas del período fundacional del partido.

\section{Conclusiones}

El análisis realizado autoriza la idea de que aquellas mujeres socialistas luchaban por la igualación de los derechos, partiendo de un modelo de ciudadanía pretendidamente universal. Pero asimismo, y este punto es quizá menos visible, la defensa de los sentimientos en la esfera pública (en la misma esfera en la cual sus compañeros imaginaban la imparcialidad de la razón) da cuenta de cierto deslizamiento por parte de aquellas mujeres frente a esta concepción liberal de la división de las esferas pública y privada división que expresa, reproduciendo, la distinción jerárquica entre los gêneros. Si la esfera pública constituye el espacio de la abstracción, la imparcialidad y la razón, propia de los hombres, la esfera privada representaría el orden de lo privado, de las determinaciones, de lo concreto, de las sensaciones, propia de las mujeres.

Se podría pensar que la idea - hegemónica entre aquellos socialistas - de individuo abstracto, racional, dueño de sí - como ejemplo de la ficción liberal del individuo moderno masculino parte de determinada forma de pensar la política. Para Juan B. Justo y sus allegados, el supuesto que organiza esa relación ideal entre economía y política se vincula con una concepción de la política fundamentalmente racional, despojada de simbologías, y que por tanto se quiere transparente, ajena a las ideologías. En este sentido, en esta posición no es sorprendente que sea exclusivamente una vía racional el fundamento de la ciudadanía 
All you need is love

universal. Por esta misma falta de atención respecto de la dimensión simbólica para la constitución de ciudadanía, tampoco es extraño que renunciaran al sostenimiento de las escuelas socialistas. ${ }^{28}$

En cambio, entre aquellas socialistas defensoras de los sentimientos como elementos articuladores en la producción de una ciudadanía moderna (fundada específicamente en los sentimientos de solidaridad y de amor) esta división público/privado, y por tanto, el ciudadano desapasionado, no era del todo aceptado, como se puede ver en relación a la "orientación práctica" de la Escuela de Morón, que intenta mezclar sensaciones con razones. Porque la crítica al individuo abstracto se basa en una concepción de la relación entre individuo, sociedad y política diferente a la anterior. Aquí el individuo aparece corporizado, sexuado. Es decir, el individuo sólo existe en tanto ser social, concreto, situado en determinada trama de relaciones de poder, y deja de ser pensado por fuera de sus determinaciones sociales (comenzando por el género).

En este sentido si bien no discuten algunas dicotomías básicas que sostienen la distribución desigual de poder entre hombres y mujeres (como la distinción entre esfera pública y privada) y luchan además por la inclusión de las mujeres en la vida pública con iguales derechos que los hombres, los "derechos del ciudadano", es esto mismo aquello que en su propia formulación permite cierto deslizamiento del modelo liberal que produce el ciudadano racional masculino. Porque sobre este modelo universalista de ciudadanía, las socialistas imprimían otros elementos - los sentimientos como articuladores del lazo social,

${ }^{28}$ Este punto podría ser el substrato teórico que posibilita que junto a otros elementos asociados (como la falta de elementos materiales para el sostén de las escuelas, y más importante aún, el significativo avance estatal en la institucionalización de la educación de masas) en 1910 los socialistas plantearan la obsolescencia de las propias escuelas. Un análisis de esta concurrencia del socialismo con las políticas educativas estatales en el período puede verse en Becerra, 2003. 
tanto en el espacio privado como en el público - que precisamente ponían en cuestión la tradicional idea de la división entre los sexos en las diferentes esferas, en especial en los inicios del siglo XX. En síntesis, si bien adscribían al modelo universalista de ciudadanía - luchando por principios universales de justicia, sin distinción de gênero - también es preciso resaltar que en ninguno de los dos ejes que sostienen este modelo, podría inscribirse a aquellas socialistas: ni en el modelo de ciudadano abstracto y racional, ni en la división por género de las esferas pública y privada. De este modo, llevaban al extremo "lo universal" de este modelo de ciudadanía, intentando superar así la trampa que la propia concepción universalista lleva en su seno.

\section{Referencias bibliograficas}

AGULHON, Maurice. Historia Vagabunda. Etnología y política en la Francia contemporánea, México, Instituto Mora, 1994.

ARICÓ, José. La Hipótesis de Justo. Bs.As., Sudamericana, 1999.

BARRANCOS, Dora. Debates sobre el sufragio en la Argentina.1890-1920. Debate Feminista, año 15, vol. 29, abril 2004, pp.293-329.

. Educación, cultura y trabajadores. Bs.As., CEAL, 1991.

BECERRA, Marina. Socialismo, Estado y nación: un análisis de la producción de hegemonía educativa estatal en Argentina. Tesis de Maestría, FLACSO, 2003.

CHABOD, Federico. La idea de nación. México, FCE, 1997.

DEL VALLE IBERLUCEA, Enrique. El divorcio. Sus fundamentos científicos. En El divorcio y la emancipación civil de la mujer, Buenos Aires, Editores Cultura y Civismo, 1919, pp.153 a 207.

El procedimiento judicial en el derecho internacional privado. Tesis Doctoral, Buenos Aires, Biblioteca Nacional, 1902.

GANDULFO, Alberto. La expansión del sistema escolar argentino. Informe estadístico. En PUIGGRÓs, A. Sociedad Civil y Estado en los orígenes del sistema educativo argentino. Buenos Aires, Galerna, Historia de la Educación Argentina, Tomo II, 1991. 
All you need is love

GONZALEZ, Manuel Meyer. Revista de Educación. Organo gremial del Magisterio de la provincia de Bs As. Año X, nº 1, 2, 3 y 4, enero y febrero de 1902.

GRAMSCI, Antonio. Notas sobre Maquiavelo, sobre politica y sobre el Estado moderno. Bs.As., Lautaro, 1962.

PATEMAN, Carole El contrato sexual. España, Anthropos, 1995.

VOLOSHINOV, V. e BAJTIN, M. El marxismo y la filosofía del lenguaje. Madrid, Alianza, 1992.

WILLIAMS, Raymond. Marxismo y literatura, Barcelona, Península, 2000.

ZAIDA LOBATO, M. (comp.) El progreso, la modernización y sus límites (1880-1916). Buenos Aires, Editorial Sudamericana, 2000.

ZIMMERMANN, Eduardo. Los liberales reformistas. La cuestión social en la Argentina (1890-1916). Buenos Aires, Sudamericana/Universidad de San Andrés, 1995. 